\title{
An exit measure construction of the total local time of super-Brownian motion
}

\author{
Jieliang Hong*
}

\begin{abstract}
We use a renormalization of the total mass of the exit measure from the complement of a small ball centered at $x \in \mathbb{R}^{d}$ for $d \leq 3$ to give a new construction of the total local time $L^{x}$ of super-Brownian motion at $x$.
\end{abstract}

Keywords: super-Brownian motion; local time; exit measure. MSC2020 subject classifications: $60 \mathrm{~J} 55 ; 60 \mathrm{G} 57 ; 60 \mathrm{~J} 68 ; 35 \mathrm{~J} 75$.

Submitted to ECP on November 9, 2020, final version accepted on June 20, 2021.

Supersedes arXiv: 2001.07269.

\section{Introduction and main results}

The local time of super-Brownian motion (SBM) has been well studied by many authors, e.g., Adler and Lewin [1], Barlow, Evans and Perkins [2], Krone [9], Sugitani [14], etc. It may be formally defined as the density function of the occupation measure of superBrownian motion. Let $M_{F}=M_{F}\left(\mathbb{R}^{d}\right)$ be the space of finite measures on $\left(\mathbb{R}^{d}, \mathfrak{B}\left(\mathbb{R}^{d}\right)\right)$ equipped with the topology of weak convergence of measures. A super-Brownian motion $X=\left(X_{t}, t \geq 0\right)$ starting at $\mu \in M_{F}$ is a continuous $M_{F}$-valued strong Markov process defined on some filtered probability space $\left(\Omega, \mathcal{F}, \mathcal{F}_{t}, P\right)$ with $X_{0}=\mu$ a.s. Write $\mu(\phi)=\int \phi(x) \mu(d x)$ for any measure $\mu$. It is well known that super-Brownian motion is the solution to the following martingale problem (see [13], II.5): For any $\phi \in C_{b}^{2}\left(\mathbb{R}^{d}\right)$,

$$
X_{t}(\phi)=X_{0}(\phi)+M_{t}(\phi)+\int_{0}^{t} X_{s}\left(\frac{\Delta}{2} \phi\right) d s,
$$

where $\left(M_{t}(\phi)\right)_{t \geq 0}$ is a continuous $\mathcal{F}_{t}$-martingale such that $M_{0}(\phi)=0$ and the quadratic variation of $M(\phi)$ is

$$
[M(\phi)]_{t}=\int_{0}^{t} X_{s}\left(\phi^{2}\right) d s .
$$

Here $C_{b}^{2}\left(\mathbb{R}^{d}\right)$ is the space of bounded functions which are twice continuously differentiable. The above martingale problem uniquely characterizes the law $\mathbb{P}_{X_{0}}$ of superBrownian motion $X$, starting from $X_{0} \in M_{F}$, on $C\left([0, \infty), M_{F}\right)$, the space of continuous functions from $[0, \infty)$ to $M_{F}$ furnished with the compact-open topology.

\footnotetext{
${ }^{*}$ University of British Columbia, Canada. E-mail: j lhong@math. ubc.ca
} 
For any $0 \leq t \leq \infty$, the occupation measure of super-Brownian motion $X$ up to time $t$ is the random measure defined by

$$
I_{t}(A)=\int_{0}^{t} X_{s}(A) d s
$$

In dimensions $d \leq 3$, the occupation measure $I_{t}$ has a density, $L_{t}^{x}$, called the local time of $X$, which satisfies

$$
I_{t}(f)=\int_{0}^{t} X_{s}(f) d s=\int_{\mathbb{R}^{d}} f(x) L_{t}^{x} d x \text { for all continuous } f \text { with compact support. }
$$

Moreover, Theorems 2 and 3 of Sugitani [14] imply that $(t, x) \mapsto L_{t}^{x}$ is continuous on $[0, \infty) \times S\left(X_{0}\right)^{c}$, where $S(\mu)=\operatorname{Supp}(\mu)$ denotes the closed support of a measure $\mu$. The extinction time of $X$ is a.s. finite (see, e.g., Chp II.5 in [13]) and so we set $L^{x}=L_{\infty}^{x}$ to be the (total) local time of $X$. We define the range, $\mathcal{R}$, of $X$ to be $\mathcal{R}=\operatorname{Supp}\left(I_{\infty}\right)$.

Now consider SBM under the canonical measure $\mathbb{N}_{x_{0}}$, which is a $\sigma$-finite measure on $C\left([0, \infty), M_{F}\right)$. If $\Xi=\sum_{i \in I} \delta_{\nu^{i}}$ is a Poisson point process on $C\left([0, \infty), M_{F}\right)$ with intensity $\mathbb{N}_{X_{0}}(d \nu)=\int \mathbb{N}_{x}(d \nu) X_{0}(d x)$, then

$$
X_{t}=\sum_{i \in I} \nu_{t}^{i}=\int \nu_{t} \Xi(d \nu), t>0
$$

has the law, $\mathbb{P}_{X_{0}}$, of a super-Brownian motion $X$ starting from $X_{0}$. We refer the readers to Theorem II.7.3(c) of [13] for more details. The global continuity of the total local time $L^{x}$ under $\mathbb{N}_{x_{0}}$ is given in [6] (see, e.g., Theorem 1.2 of the same reference). By (1.4) we may decompose the total local time $L^{x}$ under $\mathbb{P}_{X_{0}}$ as

$$
L^{x}=\sum_{i \in I} L^{x}\left(\nu^{i}\right)=\int L^{x}(\nu) \Xi(d \nu)
$$

Intuitively the total local time $L^{x}$ measures the amount of mass distributed by superBrownian motion on the singleton $x$. This mechanism is pretty similar to the exit measure from the complement of a small ball centered at $x$. To define the exit measure in an appropriate way, we first recall Le Gall's Brownian snake.

Let $\mathcal{W}=\cup_{s \geq 0} C\left([0, s], \mathbb{R}^{d}\right)$ be equipped with the natural metric (see, e.g., Chp. IV.1 of Le Gall [11]). For any $w \in \mathcal{W}$, we write $\zeta(w)=s$ if $w \in C\left([0, s], \mathbb{R}^{d}\right)$. We call $\zeta(w)$ the lifetime of $w$. The Brownian snake $W=\left(W_{t}, t \geq 0\right)$ is a $\mathcal{W}$-valued continuous strong Markov process. Let $\zeta_{t}=\zeta\left(W_{t}\right)$ and use $\hat{W}(t)=W_{t}\left(\zeta_{t}\right)$ to denote the tip of the snake at time $t$. Recall the canonical measure $\mathbb{N}_{x}$ of super-Brownian motion from above. By slightly abusing the notation, we let $\mathbb{N}_{x}$ denote the excursion measure of the snake, on $C([0, \infty), \mathcal{W})$, starting from the trivial path at $x \in \mathbb{R}^{d}$ with zero lifetime. Then we may use the Brownian snake $W$ to construct a measure-valued process $X(W)=\left(X_{t}(W), t \geq 0\right)$ under $\mathbb{N}_{x}$ such that the law of $X(W)$ under $\mathbb{N}_{x}$ is equal to that of a super-Brownian motion under the canonical measure $\mathbb{N}_{x}$, thus justifying our abusive notation. We use $X_{t}(W)$ to denote the super-Brownian motion associated with the snake $W$ instead of the integral with respect to $X_{t}$. This should be clear if one recalls that $W$ is not a function on $\mathbb{R}^{d}$ but the snake. The construction of the super-Brownian motion $X(W)$ by the snake $W$ is not important for our discussion here, and so we refer the interested readers to Theorem IV.4 of [11] for more information. If $\Xi=\sum_{j \in J} \delta_{W_{j}}$ is a Poisson point process on $\mathcal{W}$ with intensity $\mathbb{N}_{X_{0}}(d W)=\int \mathbb{N}_{x}(d W) X_{0}(d x)$, then it follows from (1.4) that

$$
X_{t}=\sum_{j \in J} X_{t}\left(W_{j}\right)=\int X_{t}(W) \Xi(d W) \text { for } t>0
$$


has the law, $\mathbb{P}_{X_{0}}$, of a super-Brownian motion $X$ starting from $X_{0}$. It also follows from (1.5) that the total local time $L^{x}$ under $\mathbb{P}_{X_{0}}$ may be decomposed as

$$
L^{x}=\sum_{j \in J} L^{x}\left(W_{j}\right)=\int L^{x}(W) \Xi(d W) .
$$

Now we turn to the exit measure. The exit measure from an open set $G$, under $\mathbb{P}_{X_{0}}$ or $\mathbb{N}_{X_{0}}$, is a random finite measure supported on $\partial G$ and is denoted by $X_{G}$ (see Chp. V of [11] for the construction of the exit measure). Intuitively $X_{G}$ represents the mass started at $X_{0}$ which is stopped at the instant it leaves $G$. We note [11] also suffices as a reference for the properties of $X_{G}$ described below. Let $B\left(x_{0}, \varepsilon\right)=B_{\varepsilon}\left(x_{0}\right)=\left\{x:\left|x-x_{0}\right|<\varepsilon\right\}$ denote an open ball centered at $x_{0} \in \mathbb{R}^{d}$ with radius $\varepsilon>0$. Define the complement of a closed ball centered at $x_{0}$ with radius $\varepsilon>0$ to be

$$
G_{\varepsilon}^{x_{0}}=G_{\varepsilon}\left(x_{0}\right)=\left\{x:\left|x-x_{0}\right|>\varepsilon\right\} \text { and let } G_{\varepsilon}=G_{\varepsilon}(0) .
$$

For any $K_{1}, K_{2}$ non-empty, set

$$
d\left(K_{1}, K_{2}\right)=\inf \left\{|x-y|: x \in K_{1}, y \in K_{2}\right\} .
$$

We assume that $x_{0} \in \mathbb{R}^{d}$ and $\varepsilon>0$ satisfy $d\left(\overline{B_{\varepsilon}\left(x_{0}\right)}, S\left(X_{0}\right)\right)>0$. In what follows we will only be considering exit measures $X_{G}$ for $G=G_{\varepsilon}^{x_{0}}$ with $x_{0} \in \mathbb{R}^{d}$ and $\varepsilon>0$ as above. Under $\mathbb{N}_{x}$ we have the range $\mathcal{R}$ of super-Brownian motion $X=X(W)$, defined by $\mathcal{R}=S\left(I_{\infty}\right)$ with $I_{\infty}$ as in (1.2), may also be written as (see, e.g., equation (8) in the proof of Theorem IV.7(iii) of [11])

$$
\mathcal{R}=\{\hat{W}(s): s \in[0, \sigma]\},
$$

where $\sigma=\sigma(W)=\inf \left\{t>0: \zeta_{t}=0\right\}>0$ is the length of the excursion path. For any $x \in G$, under $\mathbb{N}_{x}$ we may use the definition of exit measure in Chp. V of [11] to get (see also (2.3) of [8])

$X_{G}$ is a finite random measure supported on $\partial G \cap \mathcal{R}$ a.e.

The extension of (1.10) to $\mathbb{N}_{X_{0}}$ is immediate as $\mathbb{N}_{X_{0}}(d W)=\int \mathbb{N}_{x}(d W) X_{0}(d x)$. It also works under $\mathbb{P}_{X_{0}}$ as we may, equivalently, set (see, e.g., (2.23) of [12])

$$
X_{G}=\sum_{j \in J} X_{G}\left(W_{j}\right)=\int X_{G}(W) \Xi(d W),
$$

where $\Xi$ is a Poisson point process on $\mathcal{W}$ with intensity $\mathbb{N}_{X_{0}}$.

Let $d(x, K)=\inf \{|x-y|: y \in K\}$. It has been shown in Proposition 6.2(b) of [8] that for any $x \in S\left(X_{0}\right)^{c}$, under $\mathbb{N}_{X_{0}}$ or $\mathbb{P}_{X_{0}}$, the family $\left\{X_{G_{r_{0}-r}^{x}}(1), 0 \leq r<r_{0}\right\}$ with $r_{0}=d\left(x, S\left(X_{0}\right)\right) / 2$ has a càdlàg version which is a supermartingale if $d=3$; a martingale if $d=2$. Throughout the rest of the paper, we will always work with this càdlàg version. For any $\varepsilon>0$, set

$$
\psi_{0}(\varepsilon)= \begin{cases}\frac{1}{\pi} \log ^{+}(1 / \varepsilon), & \text { in } d=2, \\ \frac{1}{2 \pi} \frac{1}{\varepsilon}, & \text { in } d=3 .\end{cases}
$$

The following result gives a new construction of the total local time $L^{x}$ in terms of the local asymptotic behavior of the exit measures at $x$. This result is also useful in the construction of a boundary local time measure whose support is the topological boundary of the range of super-Brownian motion in $d=2$ and $d=3$ (see [7]).

Notation. For a collection of random variables $\left\{\xi_{t}, t \in T\right\}$, we say $\xi_{t}$ converges in measure to $\xi_{t_{0}}$ under $\mathbb{N}_{X_{0}}$ as $t \rightarrow t_{0}$ if for any $\eta>0, \mathbb{N}_{X_{0}}\left(\left|\xi_{t}-\xi_{t_{0}}\right|>\eta\right) \rightarrow 0$ as $t \rightarrow t_{0}$. The same definition applies under $\mathbb{P}_{X_{0}}$. 
Theorem 1.1. Let $d=2$ or $d=3$ and $X_{0} \in M_{F}\left(\mathbb{R}^{d}\right)$. For any $x \in S\left(X_{0}\right)^{c}$, we have

$$
X_{G_{\varepsilon}^{x}}(1) \psi_{0}(\varepsilon) \text { converges in measure to } L^{x} \text { under } \mathbb{N}_{X_{0}} \text { or } \mathbb{P}_{X_{0}} \text { as } \varepsilon \downarrow 0 \text {, }
$$

where $\psi_{0}$ is as in (1.12). Moreover, in $d=3$ the convergence holds $\mathbb{N}_{X_{0}}$-a.e. or $\mathbb{P}_{X_{0}}$-a.s. Remark 1.2. In $d=3$, the family $\mathcal{A}:=\left\{X_{G_{r_{0}-r}^{x}}(1) \psi_{0}\left(r_{0}-r\right), 0 \leq r<r_{0}\right\}$ with $r_{0}=d\left(x, S\left(X_{0}\right)\right) / 2$ is indeed a martingale (see the proof of the above theorem in Section 3). This allows us to use martingale convergence to conclude a.s. convergence in $d=3$. In $d=2$, we already know from Proposition 6.2(b) of [8] that the family $\left\{X_{G_{r_{0}-r}^{x}}(1), 0 \leq r<r_{0}\right\}$ is a martingale, and so one can check that $\mathcal{A}$ will be a submartingale in $d=2$. Whether or not a.s. convergence holds in $d=2$ remains unresolved.

\section{The special Markov property}

We will state the special Markov property for the Brownian snake from [10] that plays an essential role in our proof. We first deal with $\mathbb{N}_{X_{0}}$. Recall that we are working with exit measures $X_{G}$ for $G=G_{\varepsilon}^{x_{0}}$ with $x_{0} \in \mathbb{R}^{d}$ and $\varepsilon>0$ satisfying $d\left(\overline{B_{\varepsilon}\left(x_{0}\right)}, S\left(X_{0}\right)\right)>0$. Define

$$
\begin{gathered}
S_{G}\left(W_{u}\right)=\inf \left\{t \leq \zeta_{u}: W_{u}(t) \notin G\right\} \quad(\inf \emptyset=\infty), \\
\eta_{s}^{G}(W)=\inf \left\{t: \int_{0}^{t} 1\left(\zeta_{u} \leq S_{G}\left(W_{u}\right)\right) d u>s\right\}, \\
\mathcal{E}_{G}=\sigma\left(W_{\eta_{s}^{G}}, s \geq 0\right) \vee\left\{\mathbb{N}_{X_{0}}-\text { null sets }\right\},
\end{gathered}
$$

where $s \rightarrow W_{\eta_{s}^{G}}$ is continuous (see p. 401 of [10]). Intuitively one may think of $\mathcal{E}_{G}$ as the $\sigma$-field generated by the excursions of $W$ inside $G$. Write the open set $\left\{u: S_{G}\left(W_{u}\right)<\zeta_{u}\right\}$ as countable union of disjoint open intervals, $\cup_{i \in I}\left(a_{i}, b_{i}\right)$. Then for all $u \in\left[a_{i}, b_{i}\right]$, one notices $S_{G}\left(W_{u}\right)=S_{G}^{i}<\infty$ where $S_{G}^{i}=S_{G}\left(W_{a_{i}}\right)>0$, and we may define

$$
W_{s}^{i}(t)=W_{\left(a_{i}+s\right) \wedge b_{i}}\left(S_{G}^{i}+t\right) \text { for } 0 \leq t \leq \zeta_{\left(a_{i}+s\right) \wedge b_{i}}-S_{G}^{i} .
$$

In this way, we have $W^{i}$ are the excursions of $W$ outside $G$ for each $i \in I$. Proposition 2.3 of [10] implies that $X_{G}$ is $\mathcal{E}_{G}$-measurable and Corollary 2.8 of the same reference gives the following special Markov property:

$$
\left\{\begin{array}{l}
\text { Conditional on } \mathcal{E}_{G} \text {, the point measure } \sum_{i \in I} \delta_{W^{i}} \text { is a Poisson } \\
\text { point measure with intensity } \mathbb{N}_{X_{G}} .
\end{array}\right.
$$

Here $\mathbb{N}_{X_{G}}(d W)=\int \mathbb{N}_{x}(d W) X_{G}(d x)$ is a (random) intensity measure on the space of the snake, i.e. $C([0, \infty), \mathcal{W})$. Consider $G=G_{\varepsilon_{1}}^{x}$ and $D=G_{\varepsilon_{2}}^{x}$ with $\varepsilon_{1}>\varepsilon_{2}>0$. We can define the exit measure $X_{D}\left(W^{i}\right)$ for each $W^{i}$ following the construction of exit measure in Chapter V.1 of [11]. As in (2.6) of [8], one may conclude

$$
X_{D}=\sum_{i \in I} X_{D}\left(W^{i}\right)
$$

If $U$ is an open subset of $S\left(X_{0}\right)^{c}$, then $L_{U}$, the restriction of the total local time $L^{x}$ to $U$, is in $C(U, \mathbb{R})$ which is the set of continuous functions on $U$. Here are some consequences of (2.2) that are already proved in Proposition 2.2(a) of [8].

Proposition 2.1. For any $X_{0} \in M_{F}\left(\mathbb{R}^{d}\right)$, fix some $x \in S\left(X_{0}\right)^{c}$. Define $G_{1}=G_{\varepsilon_{1}}^{x}$ and $G_{2}=G_{\varepsilon_{2}}^{x}$ with $0<\varepsilon_{2}<\varepsilon_{1}<d\left(x, S\left(X_{0}\right)\right)$.

(i) If $\psi_{1}: C\left({\overline{G_{1}}}^{c}, \mathbb{R}\right) \rightarrow[0, \infty)$ is Borel measurable, then

$$
\mathbb{N}_{X_{0}}\left(\psi_{1}\left(L_{\overline{G_{1}}}^{c}\right) \mid \mathcal{E}_{G_{1}}\right)=\mathbb{E}_{X_{G_{1}}}\left(\psi_{1}\left(L_{\overline{G_{1}}} c\right)\right) .
$$


(ii) If $\psi_{2}: M_{F}\left(\mathbb{R}^{d}\right) \rightarrow[0, \infty)$ is Borel measurable, then

$$
\mathbb{N}_{X_{0}}\left(\psi_{2}\left(X_{G_{2}}\right) \mid \mathcal{E}_{G_{1}}\right)=\mathbb{E}_{X_{G_{1}}}\left(\psi_{2}\left(X_{G_{2}}\right)\right) .
$$

The $\sigma$-finiteness of $\mathbb{N}_{X_{0}}$ is not an issue here as we may define the above conditional expectation by, e.g., using Radon-Nikodym derivative.

We will need a version of the above under $\mathbb{P}_{X_{0}}$ as well, which follows immediately from Proposition 2.3 of [8].

Proposition 2.2. For any $X_{0} \in M_{F}\left(\mathbb{R}^{d}\right)$, fix some $x \in S\left(X_{0}\right)^{c}$. Define $G_{1}=G_{\varepsilon_{1}}^{x}$ and $G_{2}=G_{\varepsilon_{2}}^{x}$ with $0<\varepsilon_{2}<\varepsilon_{1}<d\left(x, S\left(X_{0}\right)\right)$.

(i) If $\phi_{1}: C\left({\overline{G_{1}}}^{c}, \mathbb{R}\right) \rightarrow[0, \infty)$ is Borel measurable, then

$$
\mathbb{E}_{X_{0}}\left(\phi_{1}\left(L_{\overline{G_{1}}}{ }\right)\right)=\mathbb{E}_{X_{0}}\left(\mathbb{E}_{X_{G_{1}}}\left(\phi_{1}\left(L_{\overline{G_{1}}}{ }\right)\right)\right) .
$$

(ii) If $\phi_{2}: M_{F}\left(\mathbb{R}^{d}\right) \rightarrow[0, \infty)$ is Borel measurable, then

$$
\mathbb{E}_{X_{0}}\left(\phi_{2}\left(X_{G_{2}}\right)\right)=\mathbb{E}_{X_{0}}\left(\mathbb{E}_{X_{G_{1}}}\left(\phi_{2}\left(X_{G_{2}}\right)\right)\right)
$$

\section{Construction of the total local time by exit measure}

In this section we will give the proof of Theorem 1.1. We assume throughout this section that $d=2$ or $d=3$. The Laplace transform of $L^{x}$ derived in Lemma 2.2 of [12] is given by

$$
\mathbb{E}_{X_{0}}\left(\exp \left(-\lambda L^{x}\right)\right)=\exp \left(-\int_{\mathbb{R}^{d}} V^{\lambda}(x-y) X_{0}(d y)\right)
$$

where $V^{\lambda}$ is the unique solution to

$$
\frac{\Delta V^{\lambda}}{2}=\frac{\left(V^{\lambda}\right)^{2}}{2}-\lambda \delta_{0}, \quad V^{\lambda}>0 \text { on } \mathbb{R}^{d}
$$

Here $\delta_{0}$ is the Dirac delta function and the above differential equation is interpreted in a distributional sense. One can check that $V^{\lambda}$ is radially symmetric and we may write $V^{\lambda}(|x|)$ for $V^{\lambda}(x)$. Recall $\psi_{0}$ from (1.12). It is known that (see, e.g., p. 187 of [4]) $V^{\lambda}$ is smooth in $\mathbb{R}^{d} \backslash\{0\}$, and near the origin, Lemma 8 of [3] gives that

$$
\lim _{x \rightarrow 0} \frac{V^{\lambda}(x)}{\psi_{0}(|x|)}=\lambda .
$$

Proof of Theorem 1.1. The outline for the proof is as follows: First we get some $L^{2}$ convergence, associated with $X_{G_{\varepsilon}^{x}}$ and $L^{x}$, using the Laplace transforms. Then we show that this implies the convergence in measure. When $d=3$, we prove there is an a.s. limit by the martingale arguments. It is then immediate that $L^{x}$, as the limit of convergence in measure, is in fact the a.s. limit, thus completing the proof.

We first consider the $\mathbb{N}_{X_{0}}$ case. Fix any $x \in S\left(X_{0}\right)^{c}$ and let $\delta:=d\left(x, S\left(X_{0}\right)\right)>0$. For any $\lambda>0$ and $0<\varepsilon<\delta / 2$, we have

$$
\begin{aligned}
I: & =\mathbb{N}_{X_{0}}\left(\left(\exp \left(-\lambda X_{G_{\varepsilon}^{x}}(1) \psi_{0}(\varepsilon)\right)-\exp \left(-\lambda L^{x}\right)\right)^{2}\right) \\
= & \mathbb{N}_{X_{0}}\left(\exp \left(-2 \lambda X_{G_{\varepsilon}^{x}}(1) \psi_{0}(\varepsilon)\right)+\exp \left(-2 \lambda L^{x}\right)-2 \exp \left(-\lambda X_{G_{\varepsilon}^{x}}(1) \psi_{0}(\varepsilon)\right) \exp \left(-\lambda L^{x}\right)\right) \\
= & \mathbb{N}_{X_{0}}\left(\exp \left(-2 \lambda X_{G_{\varepsilon}^{x}}(1) \psi_{0}(\varepsilon)\right)+\mathbb{E}_{X_{G_{\varepsilon}^{x}}}\left(\exp \left(-2 \lambda L^{x}\right)\right)\right. \\
& \left.\quad-2 \exp \left(-\lambda X_{G_{\varepsilon}^{x}}(1) \psi_{0}(\varepsilon)\right) \mathbb{E}_{X_{G_{\varepsilon}^{x}}}\left(\exp \left(-\lambda L^{x}\right)\right)\right)
\end{aligned}
$$


where we have used Proposition 2.1 (i) in the last equality. Apply (3.1) with $X_{0}=X_{G_{\varepsilon}^{x}}$ to get

$$
\begin{aligned}
& \mathbb{E}_{X_{G_{\varepsilon}^{x}}}\left(\exp \left(-\lambda L^{x}\right)\right)=\exp \left(-\int_{\mathbb{R}^{d}} V^{\lambda}(x-y) X_{G_{\varepsilon}^{x}}(d y)\right) \\
= & \exp \left(-\int_{\mathbb{R}^{d}} V^{\lambda}(\varepsilon) X_{G_{\varepsilon}^{x}}(d y)\right)=\exp \left(-X_{G_{\varepsilon}^{x}}(1) V^{\lambda}(\varepsilon)\right) .
\end{aligned}
$$

In the second equality we have used the fact that the exit measure $X_{G_{\varepsilon}^{x}}$ is supported on $\partial G_{\varepsilon}^{x}$ by (1.10) and then apply the radial symmetry of $V^{\lambda}$ to get $V^{\lambda}(x-y)=V^{\lambda}(|x-y|)=$ $V^{\lambda}(\varepsilon)$ for any $y \in \partial G_{\varepsilon}^{x}$. The above still holds true if we replace $\lambda$ with $2 \lambda$ in (3.5). Use the above in (3.4) to arrive at

$$
\begin{aligned}
& I=\mathbb{N}_{X_{0}}\left(\exp \left(-2 \lambda X_{G_{\varepsilon}^{x}}(1) \psi_{0}(\varepsilon)\right)+\exp \left(-X_{G_{\varepsilon}^{x}}(1) V^{2 \lambda}(\varepsilon)\right)\right. \\
& \left.\quad-2 \exp \left(-\lambda X_{G_{\varepsilon}^{x}}(1) \psi_{0}(\varepsilon)\right) \exp \left(-X_{G_{\varepsilon}^{x}}(1) V^{\lambda}(\varepsilon)\right)\right) \\
& =\mathbb{N}_{X_{0}}\left(\exp \left(-2 \lambda X_{G_{\varepsilon}^{x}}(1) \psi_{0}(\varepsilon)\right)-\exp \left(-\lambda X_{G_{\varepsilon}^{x}}(1) \psi_{0}(\varepsilon)\right) \exp \left(-X_{G_{\varepsilon}^{x}}(1) V^{\lambda}(\varepsilon)\right)\right) \\
& \quad \quad+\mathbb{N}_{X_{0}}\left(\exp \left(-X_{G_{\varepsilon}^{x}}(1) V^{2 \lambda}(\varepsilon)\right)-\exp \left(-\lambda X_{G_{\varepsilon}^{x}}(1) \psi_{0}(\varepsilon)\right) \exp \left(-X_{G_{\varepsilon}^{x}}(1) V^{\lambda}(\varepsilon)\right)\right) \\
& \quad:=I_{1}+I_{2} .
\end{aligned}
$$

We first deal with $I_{1}$.

$$
\begin{aligned}
\left|I_{1}\right| & \leq \mathbb{N}_{X_{0}}\left(\left|\exp \left(-2 \lambda X_{G_{\varepsilon}^{x}}(1) \psi_{0}(\varepsilon)\right)-\exp \left(-\left(\lambda+\frac{V^{\lambda}(\varepsilon)}{\psi_{0}(\varepsilon)}\right) X_{G_{\varepsilon}^{x}}(1) \psi_{0}(\varepsilon)\right)\right|\right) \\
& =\mathbb{N}_{X_{0}}\left(\left|X_{G_{\varepsilon}^{x}}(1) \psi_{0}(\varepsilon) \exp \left(-\lambda^{\prime}(\varepsilon) X_{G_{\varepsilon}^{x}}(1) \psi_{0}(\varepsilon)\right)\left(2 \lambda-\left(\lambda+\frac{V^{\lambda}(\varepsilon)}{\psi_{0}(\varepsilon)}\right)\right)\right|\right) \\
& \leq\left|\lambda-\frac{V^{\lambda}(\varepsilon)}{\psi_{0}(\varepsilon)}\right| \cdot \mathbb{N}_{X_{0}}\left(X_{G_{\varepsilon}^{x}}(1) \psi_{0}(\varepsilon) \exp \left(-\lambda^{\prime}(\varepsilon) X_{G_{\varepsilon}^{x}}(1) \psi_{0}(\varepsilon)\right)\right),
\end{aligned}
$$

where the second line is by the mean value theorem with $\lambda^{\prime}(\varepsilon)(\omega)$ chosen between $2 \lambda$ and $\lambda+V^{\lambda}(\varepsilon) / \psi_{0}(\varepsilon)$. When $\varepsilon>0$ is small, (3.3) implies $V^{\lambda}(\varepsilon) / \psi_{0}(\varepsilon)>\lambda / 2$, and so $\mathbb{N}_{X_{0}}$-a.e. we have $\lambda^{\prime}(\varepsilon) \geq \min \left\{2 \lambda, \lambda+V^{\lambda}(\varepsilon) / \psi_{0}(\varepsilon)\right\}>3 \lambda / 2>\lambda$. Hence (3.7) becomes

$$
\left|I_{1}\right| \leq\left|\lambda-\frac{V^{\lambda}(\varepsilon)}{\psi_{0}(\varepsilon)}\right| \cdot \mathbb{N}_{X_{0}}\left(X_{G_{\varepsilon}^{x}}(1) \psi_{0}(\varepsilon) \exp \left(-\lambda X_{G_{\varepsilon}^{x}}(1) \psi_{0}(\varepsilon)\right)\right) .
$$

Recall $\delta=d\left(x, S\left(X_{0}\right)\right)$. Define $S\left(X_{0}\right)^{>\delta / 4}=\left\{y: d\left(y, S\left(X_{0}\right)\right)>\delta / 4\right\}$ so that for any $0<\varepsilon<\delta / 2$, we have $\partial G_{\varepsilon}^{x} \subset S\left(X_{0}\right)^{>\delta / 4}$. Recall $\mathcal{R}$ from (1.9). Apply (1.10) to see for all $0<\varepsilon<\delta / 2$, we have

$$
\mathcal{R} \cap S\left(X_{0}\right)^{>\delta / 4}=\emptyset \text { implies } X_{G_{\varepsilon}^{x}}(1)=0, \quad \mathbb{N}_{X_{0}} \text {-a.e. }
$$

Use the above to get

$$
\begin{aligned}
& \mathbb{N}_{X_{0}}\left(X_{G_{\varepsilon}^{x}}(1) \psi_{0}(\varepsilon) \exp \left(-\lambda X_{G_{\varepsilon}^{x}}(1) \psi_{0}(\varepsilon)\right)\right) \\
= & \mathbb{N}_{X_{0}}\left(X_{G_{\varepsilon}^{x}}(1) \psi_{0}(\varepsilon) \exp \left(-\lambda X_{G_{\varepsilon}^{x}}(1) \psi_{0}(\varepsilon)\right) 1\left(\mathcal{R} \cap S\left(X_{0}\right)^{>\delta / 4} \neq \emptyset\right)\right) \\
\leq & \lambda^{-1} e^{-1} \mathbb{N}_{X_{0}}\left(\mathcal{R} \cap S\left(X_{0}\right)^{>\delta / 4} \neq \emptyset\right):=\lambda^{-1} e^{-1} C\left(X_{0}, \delta\right)<\infty,
\end{aligned}
$$

where the first inequality is by $x e^{-\lambda x} \leq \lambda^{-1} e^{-1}, \forall x \geq 0$. The finiteness of $C\left(X_{0}, \delta\right)$ follows from Proposition VI.2 of [11]. Hence (3.8) becomes

$$
\left|I_{1}\right| \leq\left|\lambda-\frac{V^{\lambda}(\varepsilon)}{\psi_{0}(\varepsilon)}\right| \cdot \lambda^{-1} e^{-1} C\left(X_{0}, \delta\right) \rightarrow 0 \text { as } \varepsilon \downarrow 0,
$$


where the convergence to 0 follows from (3.3).

Turning to $I_{2}$, we have

$$
\begin{aligned}
\left|I_{2}\right| & \leq \mathbb{N}_{X_{0}}\left(\left|\exp \left(-\frac{V^{2 \lambda}(\varepsilon)}{\psi_{0}(\varepsilon)} X_{G_{\varepsilon}^{x}}(1) \psi_{0}(\varepsilon)\right)-\exp \left(-\left(\lambda+\frac{V^{\lambda}(\varepsilon)}{\psi_{0}(\varepsilon)}\right) X_{G_{\varepsilon}^{x}}(1) \psi_{0}(\varepsilon)\right)\right|\right) \\
& =\mathbb{N}_{X_{0}}\left(\left|X_{G_{\varepsilon}^{x}}(1) \psi_{0}(\varepsilon) \exp \left(-\hat{\lambda}(\varepsilon) X_{G_{\varepsilon}^{x}}(1) \psi_{0}(\varepsilon)\right)\left(\frac{V^{2 \lambda}(\varepsilon)}{\psi_{0}(\varepsilon)}-\left(\lambda+\frac{V^{\lambda}(\varepsilon)}{\psi_{0}(\varepsilon)}\right)\right)\right|\right) \\
& \leq\left|\frac{V^{2 \lambda}(\varepsilon)}{\psi_{0}(\varepsilon)}-\lambda-\frac{V^{\lambda}(\varepsilon)}{\psi_{0}(\varepsilon)}\right| \cdot \mathbb{N}_{X_{0}}\left(X_{G_{\varepsilon}^{x}}(1) \psi_{0}(\varepsilon) \exp \left(-\hat{\lambda}(\varepsilon) X_{G_{\varepsilon}^{x}}(1) \psi_{0}(\varepsilon)\right)\right)
\end{aligned}
$$

where in the second line we have used the mean value theorem with $\hat{\lambda}(\varepsilon)(\omega)$ chosen between $V^{2 \lambda}(\varepsilon) / \psi_{0}(\varepsilon)$ and $\lambda+V^{\lambda}(\varepsilon) / \psi_{0}(\varepsilon)$. When $\varepsilon>0$ is small, (3.3) implies $V^{2 \lambda}(\varepsilon) / \psi_{0}(\varepsilon)>3 \lambda / 2$ and $V^{\lambda}(\varepsilon) / \psi_{0}(\varepsilon)>\lambda / 2$. So $\mathbb{N}_{X_{0}}$-a.e. we have

$$
\hat{\lambda}(\varepsilon) \geq \min \left\{\frac{V^{2 \lambda}(\varepsilon)}{\psi_{0}(\varepsilon)}, \lambda+\frac{V^{\lambda}(\varepsilon)}{\psi_{0}(\varepsilon)}\right\}>\frac{3 \lambda}{2}>\lambda .
$$

Use the above to see that (3.12) becomes

$$
\left|I_{2}\right| \leq\left|\frac{V^{2 \lambda}(\varepsilon)}{\psi_{0}(\varepsilon)}-\lambda-\frac{V^{\lambda}(\varepsilon)}{\psi_{0}(\varepsilon)}\right| \cdot \mathbb{N}_{X_{0}}\left(X_{G_{\varepsilon}^{x}}(1) \psi_{0}(\varepsilon) \exp \left(-\lambda X_{G_{\varepsilon}^{x}}(1) \psi_{0}(\varepsilon)\right)\right) .
$$

Apply (3.10) to see that

$$
\begin{aligned}
\left|I_{2}\right| & \leq\left|\frac{V^{2 \lambda}(\varepsilon)}{\psi_{0}(\varepsilon)}-\lambda-\frac{V^{\lambda}(\varepsilon)}{\psi_{0}(\varepsilon)}\right| \cdot \lambda^{-1} e^{-1} C\left(X_{0}, \delta\right) \\
& \leq\left(\left|\frac{V^{2 \lambda}(\varepsilon)}{\psi_{0}(\varepsilon)}-2 \lambda\right|+\left|\lambda-\frac{V^{\lambda}(\varepsilon)}{\psi_{0}(\varepsilon)}\right|\right) \cdot \lambda^{-1} e^{-1} C\left(X_{0}, \delta\right) \rightarrow 0 \text { as } \varepsilon \downarrow 0,
\end{aligned}
$$

where the convergence to 0 follows from (3.3).

Recall $I$ from (3.4). We may conclude from (3.11) and (3.14) that $I \rightarrow 0$ as $\varepsilon \downarrow 0$, thus giving the $L^{2}$ convergence of $\exp \left(-\lambda X_{G_{\varepsilon}^{x}}(1) \psi_{0}(\varepsilon)\right)$ to $\exp \left(-\lambda L^{x}\right)$ under $\mathbb{N}_{X_{0}}$. By Corollary 2.32 of Folland [5], for any sequence $\varepsilon_{n} \downarrow 0$, we may pick a subsequence $\varepsilon_{n_{k}} \downarrow 0$ so that

$$
\lim _{\varepsilon_{n_{k}} \downarrow 0} \exp \left(-\lambda X_{G_{\varepsilon_{n_{k}}}^{x}}(1) \psi_{0}\left(\varepsilon_{n_{k}}\right)\right)=\exp \left(-\lambda L^{x}\right), \quad \mathbb{N}_{X_{0}} \text {-a.e. }
$$

We note the arguments in Folland [5] remain valid for our setting with the $L^{2}$ convergence under the $\sigma$-finite measure $\mathbb{N}_{X_{0}}$. It is immediate from (3.15) that

$$
\lim _{\varepsilon_{n_{k}} \downarrow 0} X_{G_{\varepsilon_{n_{k}}}^{x}}(1) \psi_{0}\left(\varepsilon_{n_{k}}\right)=L^{x}, \quad \mathbb{N}_{X_{0}} \text {-a.e. }
$$

At this stage, we may not conclude the convergence in measure due to the $\sigma$-finiteness of $\mathbb{N}_{X_{0}}$. This issue could be solved by noticing that the event $\left\{X_{G_{\varepsilon}^{x}} \neq 0\right.$ or $\left.L^{x} \neq 0\right\}$ has only finite measure under $\mathbb{N}_{X_{0}}$. By using Proposition 2.1 (i), we get for any $0<\varepsilon<\delta / 2$,

$$
\begin{aligned}
\mathbb{N}_{X_{0}}\left(\left\{L^{x}>0\right\} \cap\left\{X_{G_{\varepsilon}^{x}}(1)=0\right\}\right) & =\mathbb{N}_{X_{0}}\left(1_{\left\{X_{G_{\varepsilon}^{x}}(1)=0\right\}} \mathbb{N}_{X_{0}}\left(1_{\left\{L^{x}>0\right\}} \mid \mathcal{E}_{G_{\varepsilon}^{x}}\right)\right) \\
& =\mathbb{N}_{X_{0}}\left(1_{\left\{X_{G_{\varepsilon}^{x}}(1)=0\right\}} \mathbb{E}_{X_{G_{\varepsilon}^{x}}}\left(L^{x}>0\right)\right)=0,
\end{aligned}
$$

thus giving $\mathbb{N}_{X_{0}}$-a.e. $X_{G_{\varepsilon}^{x}}(1)=0$ implies $L^{x}=0$. Together with (3.9), we get for any $0<\varepsilon<\delta / 2$,

$$
\mathcal{R} \cap S\left(X_{0}\right)^{>\delta / 4}=\emptyset \text { implies } L^{x}=0 \text { and } X_{G_{\varepsilon}^{x}}(1)=0, \quad \mathbb{N}_{X_{0}} \text {-a.e. }
$$


Therefore it follows that for any $\eta>0$,

$$
\begin{aligned}
& \mathbb{N}_{X_{0}}\left(\left|X_{G_{\varepsilon}^{x}}(1) \psi_{0}(\varepsilon)-L^{x}\right|>\eta\right) \\
& =\mathbb{N}_{X_{0}}\left(\left\{\left|X_{G_{\varepsilon}^{x}}(1) \psi_{0}(\varepsilon)-L^{x}\right|>\eta\right\} \cap\left\{\mathcal{R} \cap S\left(X_{0}\right)^{>\delta / 4} \neq \emptyset\right\}\right),
\end{aligned}
$$

and so we may work with the finite measure $\mathbb{N}_{X_{0}}\left(\cdot \cap\left\{\mathcal{R} \cap S\left(X_{0}\right)^{>\delta / 4} \neq \emptyset\right\}\right)$ when considering the convergence in measure under $\mathbb{N}_{X_{0}}$. Apply Dominated Convergence Theorem with (3.16) and (3.18) to get

$$
\lim _{\varepsilon_{n_{k}} \downarrow 0} \mathbb{N}_{X_{0}}\left(\left|X_{G_{\varepsilon_{n_{k}}}^{x}}(1) \psi_{0}\left(\varepsilon_{n_{k}}\right)-L^{x}\right|>\eta\right)=0 .
$$

Hence for any sequence $\varepsilon_{n} \downarrow 0$, there is a subsequence $\varepsilon_{n_{k}} \downarrow 0$ such that (3.19) holds, thus completing the proof of convergence in measure under $\mathbb{N}_{X_{0}}$. For the $\mathbb{P}_{X_{0}}$ case, the above arguments work in a similar and even easier way, and so we omit the details.

Now we turn to the a.s. convergence in $d=3$. For any $x \in S\left(X_{0}\right)^{c}$, set $r_{0}=\delta / 2$ where $\delta=d\left(x, S\left(X_{0}\right)\right)>0$. In $d=3$, by (6.10) of [8], for any $0<\varepsilon<r_{0}$ we have

$$
\mathbb{E}_{X_{0}}\left(X_{G_{\varepsilon}^{x}}(1)\right)=\mathbb{N}_{X_{0}}\left(X_{G_{\varepsilon}^{x}}(1)\right)=\int \frac{\varepsilon}{\left|x-x_{0}\right|} d X_{0}\left(x_{0}\right)
$$

Hence for $0<\varepsilon_{2}<\varepsilon_{1}<r_{0}$, we may apply Proposition 2.1(ii) to get

$$
\mathbb{N}_{X_{0}}\left(\frac{X_{G_{\varepsilon_{2}}^{x}}(1)}{\varepsilon_{2}} \mid \mathcal{E}_{G_{\varepsilon_{1}}^{x}}\right)=\mathbb{E}_{X_{G_{\varepsilon_{1}}}}\left(\frac{X_{G_{\varepsilon_{2}}^{x}}(1)}{\varepsilon_{2}}\right)=\frac{X_{G_{\varepsilon_{1}}^{x}}(1)}{\varepsilon_{1}},
$$

where the last equality follows by applying (3.20) with $X_{0}=X_{G_{\varepsilon_{1}}^{x}}$ and by using the fact that the exit measure $X_{G_{\varepsilon_{1}}^{x}}$ is supported on $\partial G_{\varepsilon_{1}}^{x}$ by (1.10). Recall that in $d=3$ we have $\psi_{0}(\varepsilon)=1 /(2 \pi \varepsilon)$. Use (3.21) to conclude

$$
\mathbb{N}_{X_{0}}\left(X_{G_{\varepsilon_{2}}^{x}}(1) \psi_{0}\left(\varepsilon_{2}\right) \mid \mathcal{E}_{G_{\varepsilon_{1}}^{x}}\right)=X_{G_{\varepsilon_{1}}^{x}}(1) \psi_{0}\left(\varepsilon_{1}\right),
$$

which implies $\left\{X_{G_{r_{0}-r}^{x}}(1) \psi_{0}\left(r_{0}-r\right), 0 \leq r<r_{0}\right\}$ is a nonnegative martingale. Note that we always work with the càdlàg version of $X_{G_{r_{0}-r}^{x}}$ (1) on $0 \leq r<r_{0}$. Now we may apply the martingale convergence theorem to get $\mathbb{N}_{X_{0}}$-a.e. $\lim _{r \rightarrow r_{0}} X_{G_{r_{0}-r}^{x}}(1) \psi_{0}\left(r_{0}-r\right)$ exists. Since we already have $X_{G_{\varepsilon}^{x}}(1) \psi_{0}(\varepsilon)$ converges to $L^{x}$ in measure under $\mathbb{N}_{X_{0}}$ (see also (3.16)), we conclude that $\mathbb{N}_{X_{0}}$-a.e. $\lim _{\varepsilon \downarrow 0} X_{G_{\varepsilon}^{x}}(1) \psi_{0}(\varepsilon)=L^{x}$. The case for $\mathbb{P}_{X_{0}}$ follows in a similar way.

\section{References}

[1] R. Adler and M. Lewin. Local time and Tanaka formulae for super-Brownian motion and super stable processes. Stochastic Process. Appl., 41: 45-67, (1992). MR-1162718

[2] M. Barlow, S. Evans and E. Perkins. Collision local times and measure-valued diffusions. Can. J. Math., 43: 897-938, (1991). MR-1138572

[3] H. Brezis and L. Oswald. Singular solutions for some semilinear elliptic equations, Archive Rational Mech. Anal. 99, 249-259, (1987). MR-0888452

[4] H. Brezis, L. Peletier and D. Terman. A very singular solution of the heat equation with absorption, Archive Rational Mech. Anal. 95 (1986) pp. 185-209. MR-0853963

[5] G. Folland. Real analysis: Modern techniques and their applications. Second edition. Pure and Applied Mathematics (New York). A Wiley-Interscience Publication. John Wiley\&Sons, Inc., New York, (1999). MR-1681462 
[6] J. Hong. Renormalization of local times of super-Brownian motion. Electron. J. Probab., 23 : no. 109, 1-45, (2018). MR-3878134

[7] J. Hong. On the boundary local time measure of super-Brownian motion. Electron. J. Probab., 25: no. 106, $66 \mathrm{pp}$, (2020).MR-4147519

[8] J. Hong, L. Mytnik and E. Perkins. On the topological boundary of the range of super-Brownian motion. Ann. Probab., 48: no. 3, 1168-1201, (2020). MR-4112711

[9] S. Krone. Local times for superdiffusions. Ann. Probab., 21 (b): 1599-1623, (1993). MR1235431

[10] J.F. Le Gall. The Brownian snake and solutions of $\Delta u=u^{2}$ in a domain. Probab. Theory Relat. Fields, 102: 393-432, (1995). MR-1339740

[11] J.F. Le Gall. Spatial Branching Processes, Random Snakes and Partial Differential Equations. Lectures in Mathematics, ETH, Zurich. Birkhäuser, Basel (1999). MR-1714707

[12] L. Mytnik and E. Perkins. The dimension of the boundary of super-Brownian motion. Prob. Th. Rel Fields 174: 821-885, (2019).MR-3980306

[13] E.A. Perkins. Dawson-Watanabe Superprocesses and Measure-valued Diffusions. Lectures on Probability Theory and Statistics, no. 1781, Ecole d'Eté de Probabilités de Saint Flour 1999. Springer, Berlin (2002). MR-1915445

[14] S. Sugitani. Some properties for the measure-valued branching diffusion processes. J. Math. Soc. Japan, 41:437-462, (1989). MR-0999507

Acknowledgments. This work was done as part of the author's graduate studies at the University of British Columbia. I would like to thank my supervisor, Professor Edwin Perkins, for suggesting this problem and for the helpful discussions and suggestions throughout this work. I also thank two anonymous referees for their comments and suggestions which help to improve the readability of the manuscript. 


\section{Electronic Journal of Probability Electronic Communications in Probability}

\section{Advantages of publishing in EJP-ECP}

- Very high standards

- Free for authors, free for readers

- Quick publication (no backlog)

- Secure publication $\left(\mathrm{LOCKSS}^{1}\right)$

- Easy interface (EJMS²)

\section{Economical model of EJP-ECP}

- Non profit, sponsored by $\mathrm{IMS}^{3}, \mathrm{BS}^{4}$, ProjectEuclid ${ }^{5}$

- Purely electronic

\section{Help keep the journal free and vigorous}

- Donate to the IMS open access fund ${ }^{6}$ (click here to donate!)

- Submit your best articles to EJP-ECP

- Choose EJP-ECP over for-profit journals

\footnotetext{
${ }^{1}$ LOCKSS: Lots of Copies Keep Stuff Safe http://www. lockss.org/

${ }^{2}$ EJMS: Electronic Journal Management System http://www.vtex.lt/en/ejms.html

${ }^{3}$ IMS: Institute of Mathematical Statistics http://www.imstat.org/

${ }^{4}$ BS: Bernoulli Society http://www. bernoulli-society.org/

${ }^{5}$ Project Euclid: https://projecteuclid.org/

${ }^{6}$ IMS Open Access Fund: http://www.imstat.org/publications/open.htm
} 